\title{
Revised list of the genus Peltigera (lichenized Ascomycotina) and peltigericolous fungi from Lithuania
}

\author{
JURGA MOTIEJŪNAITE ${ }^{1}$ and JOLANTA MIADLIKOWSKA ${ }^{2}$ \\ ${ }^{1}$ Institute of Botany \\ Žaliuju ě̆eru 49, LT.2021 Vilnius, Lithuania \\ ${ }^{2}$ Department of Plant Ecology and Nature Protection, University of Gdansk \\ Al. Legionów 9, PL-80441 Gdańsk, Poland
}

Motiejü na it é J., M i i d $\mathbf{l}$ ik ow s k a J.: Revised list of the genus Peltigera (tichenized Ascomycotina) and peltigericolous fungi from Lithuania. Acta Mycol. 33 (2): 309-316, 1998.

Fifteen species of Peltigera genus and 5 species of Peltigera inhabiting fungi are reported from Lithuania. Three Peitigera species ( $P$. degenit, $P$. hymenina and $P$. ponojensis) and all peltigericolous fungi are new to the studied area. One earlier recorded species - Peltigera collina, is not included in the present list. Distribution maps for all Peftigera species are provided.

Key words: lichens, Peifigera, lichenicolous fungi, distribution, Lithuania.

\section{INTRODUCTION}

The present paper is the first one dealing in details with genus Peligera in Lithuania. In the checklist of Lithuanian lichens ( $\mathrm{R} \mathrm{u} \mathrm{d} \mathrm{z} \mathrm{i} \mathrm{n} \mathrm{k} \mathrm{a} \mathrm{i} \mathrm{t} \mathrm{é}$ 1982) 11 species and 3 varieties of the genus Pelligera are included. In some later works two more species were added to the list: $P$. neckeri (M o t i ejüna i té 1988) and $P$. membranacea (M ot i e jūna i é 1995). The present list includes 3 new for Lithuanian flora Peltigera species: $P$. degenii, $P$. hymenina and $P$. ponojensis.

This paper deals with modern as well as old herbarium data. Literature data where no herbarium material was available was not treated, for critical revision of known herbarium material showed that considerable part of it does not agree with original reports. Following this principle Peltigera collina (Ach.) Schrader was not included in the present list, the only record of this species in Lithuania being the manuscript by $\mathrm{P}$ a b r é ž a (1843), which lacks whatever collecting locality indications or herbarium voucher. 
Distribution of lichenicolous fungi in Lithuania was not studied before (only one species, Muelerella pigmaea (Körb.) D. Hawksw. known from earlier reports (M o t i e jū na i té 1990). Thus all five species of peltigericolous fungi, are new to the studied area, however random was their study.

The data on soils and climate of Lithuania are presented by $\mathrm{B}$ a $\mathrm{S}$ a $\perp$ yk as (1958), phytogeography - N a t k e vića it é-I va na us kien é (1983). The area under study in the present paper includes whole territory of Lithuania, however, more field work is still required, especially in some central and northern districts.

\section{MATERIAL AND METHODS}

This study is based on modern as well as old herbarium collections from Lithuania which authors of the paper were able to track in various herbaria. Most of the modern material is deposited in BILAS (Botanical Institute, Vilnius, Lithuania) and WI (Vilnius University, Dept. of Botany and Genetics, Lithuania), some duplicates of it are in UGDA (Gdanisk University, Poland), and $\mathbf{H}$ (Botanical Museum, University of Helsinki, Finland). Old collections were studied in BILAS, WI, H and one specimen from KRAM-L (Herbarium of the Academy of Sciences, Kraków, Poland). The names of Peltigera species are cited after V it i k a in e n (1994), of lichenicolous fungi - after Santes s on (1993) and $\mathrm{H}$ aw k sworth (1981). The species were determined on the base of their morphological characters, some critical specimens were revised by Dr. Orvo $\mathrm{V}$ it ik a in en.

\section{LIST OF PELTIGERA SPECIES}

Abbreviations: Bač - Bačiauskaité; Mich - Michalski; Min - Minkevičius; Mo - Motiejūnaité; Pab - Pabréža; Rudz - Rudzinskaité.

P. aphthosa (L.) Willd. (Fig. 1). On soil and stones among the mosses. Specimens examed: W1: Palanga, 18..., Pab; Trakai: Rekalnis, 1930, Mich.

P. canina (L.) Willd. (Fig. 2). On soil among and on mosses, on decaying tree stumps and trunk bases, on moss-covered concrete, in forests and meadows. Often associated with other terricolous lichen species: Cetraria aculeata, C. ericetorum, C. islandica, Cladonia arbuscula, C. chlorophaea, C. crispata, C. fimbriata, C. furcata, C. glauca, C. phyllophora, C. scabriuscula, and most of Peltigera species found at present. Common in Lithuania. 
Specimens examed: BILAS: Neringa, Juodkranté, 1984, Mo; Sakiai: Vaidgiris forest, 1987. Mo; Kretinga: Darbénai, 1986, 1987, Mo: Salantai, 1987, Mo: Varéna: Samūnišcés, 1990. Mo; Pancvéžys: Zalioji giria forest, 1992, Mo; Jurbarkas: Šiliné, 1987, Mo; Molétai Videniskiai, 1988, Mo; Švenčionys: Pabradé military forestry, 1994, 1995, Mo; Klaipéda: Kairiai military forestry, 1994, 1995, Mo; Nemirseta military forestry, 1994, 1995, Mo: Marijampolé: Bagotoji military forestry, 1995, Mo; Palanga, 1988, Adamonyté; Trakai: Kazokiskis forest, 1994, Dringelis; Šalěininkai: Rudninkai, 1962, Min; WI: Trakai, 1929. anonymous; Vilnius: Karoliniškés, 1932. Mich; Antaviliai, 1956, Min; Zarasai, 1935, Bač: Anykščiai, 1960, Rudz; Trakai: Tolkiškés, 1978, Vaiciulyté; Trakai-Semeliškés, 1960, Snarskis; Neringa, Juodkrantẻ, 1976, Rudz; Varéna: Valkininkai, 1977, Rudz; Puvočiai, 1985. Grigaité; Palanga, 18.... Pab, 1947, Snarskis; H: Kaunas, Veršviai, Freda, 1931, Linkola.

P. degenii Gyeln. (Fig. 3). On soil and mosses in forest.

Specimen examed: WI: Trakai: Varninkéliai forest, 1930, Mich.

P. didactyla (With.) J. R. Laundon var. didactyia (Fig. 4). On soil in pine and pine-birch forests and dry meadows, in recently disturbed places - gravel-pits, forest quarter lines, on mosses, covering stonewalls, rarely - on old decaying polypore fruit-bodies. Often associated with other terricolous lichen species: Cetraria aculeata, C. chlorophaea, C. fimbriata, C. furcata, C. glauca, C. phyllophora, C. scabriuscula, other Peltigera species: P. canina, $P$. hymenina, $P$. ponojensis, $P$. rufescens. When growing on decaying tree stumps and old polypores are associated with Cladonia coniocraea, C. fimbriata, Hypogymnia physodes. On disturbed and burned soil - with Placynthiella uliginosa and Stereocaulon condensatum. Common in Lithuania.

Specimens examed: BILAS: Šakiai: Vaidgiris forest, 1987, Mo; Neringa, Juodkranté forestry, 1987, Mo; Jurbarkas: Siliné, 1987, Mo; Jurbarkas forest, 1987, Mo; Ignalina: Motiejūnai, 1989, Mo: Varéna: Marcinkonys, 1992, Mo; Panevérys: Žalioji giria forest, 1992, Mo; Vilnius, Verkiai park, 1987, Mo; Marijampolé: Bagotoji military forestry, 1995, Mo; Klaipéda: Nemirseta military forestry, 1994, 1995, Mo; Jonava: Gaið̌iunai military forestry, 1994, Mo; Kaisiadorys: Neprékšta, 1994, Mo; Švenêtonys: Lakaja forest, 1994, Mo; Salčininkai: Rüdninkai military forestry, 1995, Mo; Utena: Aukštaitija National Park, Vaišnorišké, 1995, Mo; Trakai: Kazokiškis forest, 1994 Dringelis; Tauragé: Viešvilé reserve, 1994, Usclienć; WI: Vilnius: Karoliniškés, 1931, Mich; Zalieji eźcrai, 1961, Rudz; Salčininkai: Jašiũnai, 1961. Min; Zarasai: Turmantas-Dūkštas, 1963, Min.

- var. extenuata (Nyl. ex Vain.) Goffinet et Hastings (Fig. 4). On soil in meadow, on mosses on path side in pine forest. Rare, new to Lithuania. Specimens examed: BILAS: Varéna: Zervynos forestry, 1993, Mo; Švenèionys: Pabrade military forestry, 1994, Mo.

P. hymenina (Ach.) Delise (Fig. 5). On mosses and fallen leaves in meadows, heathlands and birch forests. Often associated with other Peltigera species: $P$. canina, $P$. didactyla, $P$. rufescens and terricolous Cladonia fimbriata and C. furcata. Scattered. New to Lithuania.

Specimens examed: BILAS: Kretinga: Darbénai, 1987, Mot; Jurbarkas: Jūrava forestry, 1987, Mo; Neringa, Juodkranté forestry, 1987, 1988, Mo; Panevéžys: Zalioji giria forest, 1992, Mo; Klaipéda: Nemirseta military forestry, 1995, Mo; Tauragé: Viešvilé reserve, 1994, Useliené; WI: Palanga, 18..., Pab; Plungé: Ringé forest, 1938, Bač; Kuršiu spit, 1970, 1976, Rudz. 

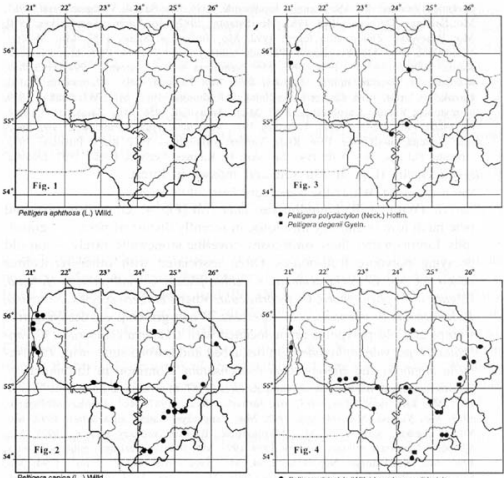

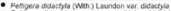

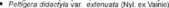
Geffret 8 Hasifings

P. lepidophora (Vain.) Bitter (Fig. 6). On soil-covered boulder.

Specimen examed: WI: Kretinga: Slučkai, 1938, Min.

P. malacea (Ach.) Funck (Fig. 7). On soil and mosses in heathlands, pine and pine-birch forests. Often associated with $P$. canina, $P$. rufescens, Cetraria ericetorum and Cladonia crispata. Rather common.

Specimens examed: BILAS: Šakiai: Vaidgiris forest, 1987, Mo: Neringa, Juodkranté, 1987, Mo; Varéna: Skroblus reserve, 1994, Mo; Kapiniškès forest, 1992, Mo; Jonava: Gaißiūnai military forestry, 1994, Mo Šalcininkai: Jašiunai forest, 1986, Mo; Rũdninkai military forestry, 1994, Mo; Švenčionys: Pabradé military forestry, 1994, 1995. Mo; Trakai: Kazokiškis forest, 1994, Dringelis; WI: Trakai: Užtrakis forest, 1948, Min; (S̄venčionys: Pabradé, 1962, Min; Vilnius: Ružiškés, 1961, Min; Nemenčiné, 1961. Rudz; Ignalina: Baluošas, 1979. Rudz; Lazdijai: Statišké forest, 1981, Ambrozaitiené: Kuršiu spit, 1968, Rudz; Varena: Valkininkai, 1977, Rudz; Puvočiai, 1985, Baltrūnaité; Kaiß̌iadorys: Gastilonys, 1957. Lalyté: Zarasai: Salakas, 1961, Rudz. 
P. membranacea (Ach.) Nyl. (Fig. 8). On soil in pine and pine-birch forests, on mossy tree trunks in humid shaded sites. When growing on soil is associated with $P$. polydactylon, $P$. rufescens, Cladonia scabriuscula. Scattered.

Specimens examed: BILAS: Kretinga: Darbénai, 1986, Mo; Neringa, Juodkranté forestry, 1987. Mo; Klaipèda: Nemirseta military forestry, 1994, Mo; Varéna: Zervynos, 1995. Grigaité; Tauragé: Viẻ̌vilé reserve, 1994, Useliené; WI: Palanga, 18.... Pab; Vilnius, Karoliniškés, 1931, Mich.

P. neckeri Hepp ex Müll. Arg. (Fig. 9). On mosses in grasslands, pine forests and forest edges, rarely on mossy boulders. Often associated with $P$. rufescens and Cladonia furcata. Scattered.

Specimens examed: BILAS: Kelmé: Kurtuvénai Regional Park, 1994. Prigodina; Lazdijai: Meteliai, 1986, Mo; Kretinga: Salantai, 1986, Mo; Mauhiai forest, 1987, Mo; Neringa, Juodkranté, 1987, Mo: Varéna: Puvočiai, 1993, 1995, Mo; Vilnius, Kairénai, 1993, Junenaité.
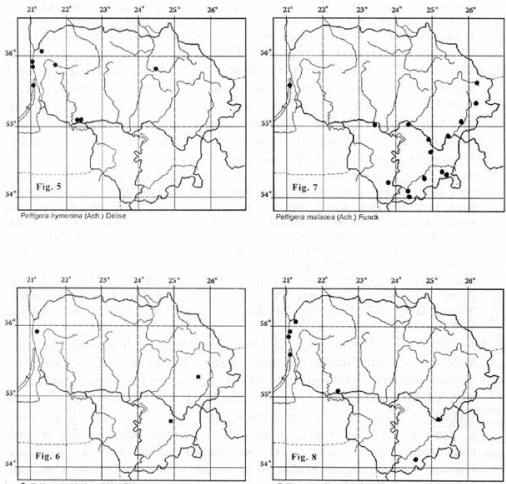
P. polydactylon (Neck.) Hoffm. (Fig. 3). On soil and mosses in grasslands and forest edges, on decaying tree trunks in shaded humid forests. When growing on soil often is associated with $P$. rufescens and Cladonia scabriuscula. Rare.

Specimens examed: BILAS: Kretinga, Salantai, 1986, Mo; Panevézys: Žalioji giria forest, 1992, Mo; Varćna: Puvočiai, 1993, Mo; Klaipéda: Nemirseta military forestry, 1994, Mo; Jonava: Gaižiunai military forestry, 1994, Mo; H: Kaunas: Raudondvaris, 1931, Linkola.

P. ponojensis Gyeln. (Fig. 10). On soil in grassland, on carth bank at forest edge, on mosses in pine-birch and spruce forests. Associated with other terricolous lichens: Cetraria aculeata, C. islandica, Cladonia arbuscula, C. chlorophaea, $C$. furcata, C. phyllophora, C. scabriuscula, other Peltigera species: $P$. canina, $P$ didactyla, $P$. praetexiata, $P$, rufescens. Scattered. New to Lithuania.

Specimens examed: BILAS: Šveněionys: Pabradé military forestry, 1994, Mo; Klaipéda: Nemirseta military forestry, 1995, Mo, Marijampolé: Bagotoji military forestry, 1995, Mo; Varéna: Puročiai, 1993, Cernevskaja; W1: Trakai: Rékalnis, 1932, Mich; Užrakis forest, 1961, Min; Ignalina: Ažvinčiu forest, 1979, Rudz.

P. praetextata (Flörke ex Sommerf.) Zopf. (Fig. 11). On decaying logs and tree-stumps, tree bases, mossy boulders, more rarely on soil in shady, humid sites. When growing on soil is associated with $P$. panojensis, on tree trunk base - with Cladonia cenotea, $C$. coniocraca, $C$. cornuta, C. crispata, C. digitata, C. fimbriata, C. macilenta, C. achrochlora, Hypogymnia physodes, Vulpicidia pinastri. Common.

Specimens examed: BILAS: Alytus: Vidzgiris, 1985, Mo; Kédainiai: Petkunai forest, 1989. Mo; Dotnuva forest, 1991, Kovéra, Pakruojis forest, 1991, Mo; Šiauliai: Gubernija forest, 1991, Mo; Biržai forest, 1992, Mo; Marijampolé: Bukıa forest, 1986, Mo; Bagotoji military forestry, 1995, Mo; Kretinga: Grôslaukis forest, 1987, Mo; Mažuc̉aa forest, 1987, Mo; Varniskis forest, 1987, Mo; Vilnius: Verkiai park, 1987, Mo; Čekoniškés, 1986, Mo; Zalieji ežerai, 1993, Lapeĺ; Neringa, Juodkranté, 1988, Mo; Švenčionys: Pakrctuoné, 1995, Mo: WI: Vilnius: Karoliniškés, 1930, 1931, Mich; Verkiai, 1930, Mich; Žalicji ežerai, 1961, Min; Trakai: Naujieji Trakai, Rékalnis, 1930, Mich; Tolkişkes, 1978, Vaičiulytć: Zarasai: Antalicpté, 1995, Prigodina; KRAM-L: Raseiniai: Blinstrubiškiai, 1909, Janczewski; H: Kaunas: Freda, Jiesia, Veršviai. Raudondvaris, 1931, Linkola.

P. rufescens (Weiss) Humb. (Fig. 12). On soil in dry, most frequently insolated habitats - pine forests, meadows, dunes, heathlands, more rarely on mossy boulders and concrete blocks or deciduous tree bases. Associated with all Peltigera species found at present, variety of terricolous Cladonia species: C. cariosa, C. cervicornis ssp. verticillata, C. chlorophaea, C. fimbriata, C. furcata, C. ramulosa, C. scabriuscula, as well as with terricolous Cetraria species: C. aculeata, C. ericetorum, C islandica. On soil in calcareous heaths it is found with together with Bacidia bagliettoana. Common. 

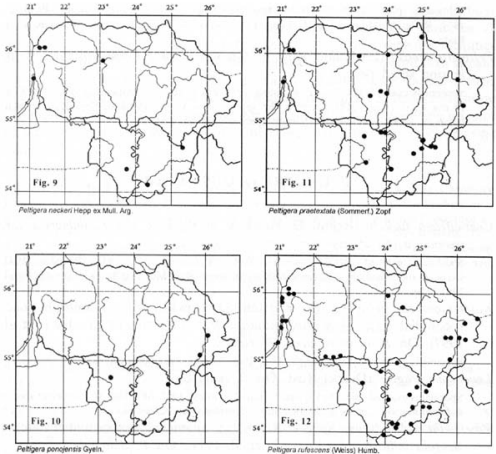

Specimens examed: BILAS: Neringa, Juodkranté forestry, 1987, Mo; Utena: Minčia, 1984, Mo; Aukštaitija National Park, Vaišniunai, 1995, Mo; Kretinga: Reivai forest, 1960, Jaskonis: Tubausiai, 1987, Mo; Darbénai, 1987, Mo; Mažučiai forest, 1987, Mo; Pakruojis: Rozalimas forest, 1991, Mo; Varéna: Kapiniskiai forest, 1992, Mo; Merkiné. 1992, Mo; Linelis lake, 1986, Mo; Rudnia forestry, 1993. Mo; Puvočiai, 1993, 1995. Mo: Klaipéda: Kairiai military forestry, 1995, Mo; Nemirseta military forestry, 1994. 1995, Mo; S̄venćionys: Pabradé military forestry, 1994, 1995, Mo: Marijampolé: Bagotoji military forestry, 1995, Mo: Salčininkai: Rüdninkai military forestry, 1994, Mo; Jonava: Gaižiunai military forestry, 1994, Mo; Panevéžys: Žalioji giria forest, 1992, Mo; Jurbarkas: Siliné, 1987, Mo: Jurbarkas forest. 1987, Mo; Jurava forestry, 1987. Mo: Molétai: Rudesélis, 1990, Mo; Videniškiai, 1988, Mo; Zarasai: Tilž́, 1986, Mo; Ignalina, 1986, Mo; Alytus, 1986, Mo; Vilnius, Verkiai, 1984, Mo; Lazdijai: Gerdašiai, 1987, Treigiene; Trakai, 1961, Jaskonis; Palanga, 1988, Adamonyte; Vilnius, Visoriai, 1992, Adamonyte; Tauragé: Vieđvilé reserve, 1991, Lapelé; WI: Kaunas: Kleboniškis, 1935, 1937, Klemas: Panemuné, 1936, Klemas; Palanga, 18..., Pab; Trakai: Rékalnis, 1930, 1932, Mich; Varninkéliai forest, 1961, Min; Tolkiškés, 1978, Vaičiulyté; Molétai: Baltieji Lakajai, 1962, Min; Neringa: Pervalka, 1962, Abisalaité \& Min; Nida, 1944, Bač; Karčiama hill. 1976, Rudz; Vilnius: Baltoji Voke, 1946, Bač, Vilnius: Antakalnis, 1985, Sirvinskaité: 
Belmontas park, 1985, Venskuté: Ignalina: Palušé, 1973, Rudz; Varéna: Pičiupiai, 1977, Rudz; Valkininkai, 1977, Rudz; Puvoèiai, 1984, Kiškyté; Zarasai: Salakas, 1961, Rudz.

Peltigera venosa (L.) Hoffm. (Fig. 6). On soil in pine forest, on earth bank among small pebbles.

Specimens examed: WI: Trakai: Rékalnis, 1930, Mich; Molétai: Balticji Lakajai, 1928, Min. M ow s z o w i z (1957) reports the specics from Vilnius (Verkiai, Grybiskés) as well, however, locality of these herbarium specimens is not known.

\section{PELTIGERICOLOUS FUNGI}

Corticifraga fuckelii (Rehm) D. Hawksw. et R. Sant. On P. didactyla var. extenuata, on $P$. rufescens, on $P$. ponojensis.

Specimens examed: BILAS: Varèna: Zervynos forestry, 1987, Mo; Puvoc̀iai, 1993, Cernevskaja; Svenéionys: Pabradé military forestry, 1994, Mo; WI: Trakai: Uãtrakis forest, 1961, Min.

Karsteniomyces peltigerae (P. Karsten) D. Hawksw. (the anamorph (macroconidial state) of Scutula miliaris (Wallr.) Trevis. (T r i e b e I et al. 1997). On $P$. membranacea, $P$. canina.

Specimens examed: W1: Palanga, 18..., Pab; Trakai, 1929, anonymous. Leocarpus fragilis (Dicks.) Rost. On P. malacea.

Specimens examed: BILAS: Varéna: Kapiniškiai forest, 1992, Mo. This Myxomycetes species is fortuitous on lichens and can not be considered as lichenicolous.

Libertiella malmedyensis Speg. et Roum. (the anamorph (mesoconidial state) of

Scutula miliaris (T r i e be 1 et al. 1977). On P. didactyla.

Specimens examed: BILAS: Tauragè: Vießvilé reserve, 1994, Uselicné. Nectria lecanodes Cesati On $P$. didactyla var. extenuata.

Specimens examed: BILAS: Varéna: Zervynos military forestry, 1987, Mo. Pronectria erythrinella (Nyl.) Lowen On $P$. didactyla.

Specimens examed: WI: Vilnius, Žalicji ežcrai, 1961, Rudz

\section{FINAL REMARKS}

Genus Peltigera in flora of Lithuania can be considered rather rich. Of 29 species in the genus, known in Europe 15 are reported from the studied area. Taking in consideration, that 11 of all European species according to distributional data in $\mathrm{V} \mathrm{it} \mathrm{i} \mathrm{k} \mathrm{a} \mathrm{i} \mathrm{n} \mathrm{e} \mathrm{n} \mathrm{(1994)} \mathrm{are} \mathrm{very} \mathrm{unlikely} \mathrm{to} \mathrm{be} \mathrm{found}$ in Lithuania, the species composition of Lithuanian Peltigera is rather complete. 
As species distribution still requires studies, not much can be said about spreading of some species in the studied area. Especially this concerns $P$. aphthosa, $P$. venosa, $P$. lepidophora, $P$. degenii - of which very few localities are known, all of them - from old collections. Most widely spread taxa appear to be $P$. rufescens, $P$. canina, $P$. praetextata, $P$. didactyla. Two species in their distribution pattern show rather different area preferences: $P$. malacea is more common in eastern, more continental districts, meanwhile $P$. hymenina was not found in the eastern part of Lithuania, preferring territory with marine influence.

AKNOWLEDGEMENTS. J. Motiejunaité is obliged to the Centre for International Mobility, Helsinki and Open Lithuania Foundation for financial support to work at the Herbarium of Botanical Museum, University of Helsinki in 1996 and J. Miądlikowska to the Committee for Scientific Research in Poland (KBN) for financial support (grant no 1170/PO4/96/10). Both authors want to express their sincere gratitude to Dr. Orvo Vitikainen, Botanical Museum, Helsinki University, for revision of critical Poligera specimens and Dr. Grażina Adamonyté, Institute of Botany, Vilnius, for determination of Leocarpas species.

\section{REFERENCES}

B as a ly k as A. (ed.) 1958. Lietuvos TSR fizine geografija. 1. Vilnius, 504 p., 194 fig. Haw ksworth D. L. 1981. The lichenicolous Coelomyeetes. Bull. Br. Mus, nat. Hist. (Bot.) 9 (1) $1-98$.

Moticjünaité J. 1988. New species of lichens in Lithuania. Tezisy dokladov Xl simpoziuma mikologoy 1 lichenologov Pribaltijskich respublik 1 Belorussii. Riga: 149-152. (In Russian).

M o t i e jü n a i t è J. 1990 . Analysis of lichen flora of western southwestern parts of Lithuania. Candidate of sciences thesis, synopsis, Vilnius. Institute of Botany. 19 p. (In Russian).

M o t i e jũ na it é J. 1995. New or interesting lichen species for Lithuania. Ekologija 4: 16-22

M owszowicz J. 1957. Porosty. Conspectus forae Vilnensis. I. Wstęp i flora zarodnikowa okolic Wilna. Lódzkie Tow. Nauk., Wydz. I11, Lódź 47: 131-143.

Natkeviéa ité-Ivana usk i n é M. 1983. Botanine geografija ir fitocenologijos pag. rindai. Vilnius. 280 p., 99 fig.

$\mathrm{P}$ a b r c ž a A. 1843. Tayslos augumyyinis, koremy taalpynas auginys ligsziol raastosys teyp mussosys kayp nckorios swetszaalys so prijungymo ablandy prajoowa weykala yr sorraszima waardu paprastuuju tcyp żemaytyszku, kaypo pruusyszku, laatwyszku yr waardu tayslyniu žemaytyszku so latiinyszkays. National Library of M. Mažvydas, Vilnius, Lithuania (mscr.). $980 \mathrm{p}$.

Rudzinskafté T, 1982. Kerpiu floros tyrimu Lietuvoje 1791-1979 m. suvestiniai duomenys. Biologija 20: 110-151.

S a n t es s o n R. 1993. The lichens and lichenicolous fungi of Sweden and Norway, Lund. $240 \mathrm{p}$.

Triebel D, Wedin M, Ra mbold G, 1997. The genus Scurula (lichenicolous ascomycetes, Lecanorales): species on the Peltigera canina and $P$, horizantalis groups. Symb.Bot. Upps. $32(1): 323-337$.

$\mathrm{V}$ i t ik a i e n O. 1994. Taxonomic revision of Peltigera (tichenized Ascomycosina) in Europe. Acta Bot. Fennica 152: 1-96. 
Zrewidowana lista gatunków z rodzaju Peltigera (Ascomycotina lichenisata) i grzybów występujących na plechach gatunków z rodzaju Peltigera na Litwic

Streszczenie

Zrewidowano materialy zielnikowe porostów z rodzaju Peltigera na Litwie, zdeponowane glównie w herbariach wileńskich (BILAS i WI). Przyjmując współczesne koncepcje gatunków okazy identyfikowano na podstawie cech morfologicznych. Podano 15 taksonów Peltigera, z k tórych trzy: $P$. degenii, $P$. hymenina i $P$. ponojensis, stwierdzono po raz pierwszy na Litwie. Nie potwierdzono występowania $P$. collina, gatunku uwzględniancgo we weześniejszych publikacjach z lego obszaru. Zamieszczono mapy rozmicszcrenia poszczcgólnych gatunków. Zidentyfikowano ponadto pięć gatunków grzybów rosnących na plechach Peltigera; żaden z nich nie był dotychezas notowany na Litwic. 